\title{
Protection of Fecal Microbiota Transplantation in a Mouse Model of Multiple Sclerosis
}

\author{
Kanglan Li $\mathbb{D}^{1},{ }^{1}$ Shouchao Wei, ${ }^{1}$ Li Hu, ${ }^{1,2}$ Xiaojian Yin, ${ }^{1}$ Yingren Mai, ${ }^{1}$ Chunmei Jiang, \\ Xiaoping Peng, ${ }^{1}$ Xingxing Cao, ${ }^{1}$ Zhongkai Huang, ${ }^{3}$ Haihong Zhou, ${ }^{1}$ Guoda Ma $\left({ }^{\circ},{ }^{1}\right.$ \\ Zhou Liu $\mathbb{D}^{1}{ }^{1}$ Huiliang $\mathrm{Li} \mathbb{D}^{4},{ }^{4}$ and Bin Zhao $\mathbb{D}^{1}$ \\ ${ }^{1}$ Guangdong Key Laboratory of Age-Related Cardiac and Cerebral Diseases, Institute of Neurology, Department of Neurology, \\ Affiliated Hospital of Guangdong Medical University, Zhanjiang, Guangdong 524001, China \\ ${ }^{2}$ Department of Histology and Embryology, Guangdong Medical University, Zhanjiang, China \\ ${ }^{3}$ Department of Neurosurgery, Affiliated Hospital of Guangdong Medical University, Guangdong Medical University, \\ Zhanjiang, China \\ ${ }^{4}$ Wolfson Institute for Biomedical Research, University College London, London WC1E 6BT, UK
}

Correspondence should be addressed to Zhou Liu; liuzhou102@126.com, Huiliang Li; huiliang.li@ucl.ac.uk, and Bin Zhao; binzhaoe@163.com

Received 17 April 2020; Revised 18 June 2020; Accepted 13 July 2020; Published 5 August 2020

Guest Editor: Hongmei Jiang

Copyright (c) 2020 Kanglan Li et al. This is an open access article distributed under the Creative Commons Attribution License, which permits unrestricted use, distribution, and reproduction in any medium, provided the original work is properly cited.

\begin{abstract}
Given the growing evidence of a link between gut microbiota (GM) dysbiosis and multiple sclerosis (MS), fecal microbiota transplantation (FMT), aimed at rebuilding GM, has been proposed as a new therapeutic approach to MS treatment. To evaluate the viability of FMT for MS treatment and its impact on MS pathology, we tested FMT in mice with experimental autoimmune encephalomyelitis (EAE), a mouse model of MS. We provide evidence that FMT can rectify altered GM to some extent with a therapeutic effect on EAE. We also found that FMT led to reduced activation of microglia and astrocytes and conferred protection on the blood-brain barrier (BBB), myelin, and axons in EAE. Taken together, our data suggest that FMT, as a GM-based therapy, has the potential to be an effective treatment for MS.
\end{abstract}

\section{Introduction}

Multiple sclerosis (MS) commonly occurs as a progressive central nervous system (CNS) disease, characterized by inflammation, demyelination, and axonal loss in the brain and spinal cord [1]. T cell-mediated inflammatory pathology and genetic factors are closely involved in the development of MS, causing damage to myelin sheaths surrounding neuronal axons and accumulation of neurological deficits [2-4]. Environmental factors also play a driving role in the pathogenesis of MS, such as geographical latitude, vitamin D3 deficiency, early life obesity, passive smoking, Epstein-Barr virus infection, dietary habits (especially high salt and fat diet), stress, and gut microbiota (GM) [5]. Study has shown that transplanting the intestinal microbiota of autism spectrum disorder patients into germ-free mice and that colonization of the microbiota induced typical autism spectrum disorder behaviors [6]. Germ-free mice developed severe MS symptoms after microbiota transplants from MS patients compared with transplanted healthy controls [7]. MS patient-derived microbiota resulted in a spontaneous EAE in a transgenic mouse model [8]. Human bacteria was transferred to mice can be detected and was a shift of the microbiota over time [9]. Notably, accumulating new evidence points to a link between altered intestinal microbiota and MS pathogenesis [10-15].

Investigation of GM has revealed significantly altered abundances of certain bacterial genera in MS patients compared to healthy controls [16]. Moreover, germ-free mice prove to be resistant to experimental autoimmune encephalomyelitis (EAE), a commonly used animal model of MS 
$[17,18]$. Together, these studies imply a causal association between GM and MS. Although the mechanisms underlying the role of GM in MS are still elusive, GM-based therapeutic strategies hold the promise of new treatments for MS.

Fecal microbiota transplantation (FMT) appears to be an effective treatment for Clostridium difficile infection and inflammatory bowel syndrome, able to restore GM diversity to some extent $[19,20]$. Some case reports suggest that FMT may help improve symptoms of epilepsy and Parkinson's disease [21, 22]. Interestingly, one study found that after FMT treatment for constipation, three wheelchair-bound MS patients had so dramatic improvement in neurological symptoms that they regained the ability to walk unassisted [23]. Therefore, FMT has the potential to be an innovative therapy for MS. Here, we evaluate the effect of FMT on EAE and explore possible mechanisms behind it. Our data reveal that FMT can improve the clinical outcome of EAE by modulating GM, reducing glial inflammatory response and conferring protection on the blood-brain barrier (BBB), myelin, and axons.

\section{Materials and Methods}

2.1. Animals. Four- to five-week-old female C57BL/6 mice were purchased from Guangdong Medical Laboratory Animal Center and raised in pathogen-free conditions in an animal facility at Guangdong Medical University (GMU). Mice were allowed 1 week's habituation before being used for experiments. Animal care and all procedures complied with the guidelines of GMU Experimental Animal Ethics Committee and national laws and regulations of China for use of animals in biomedical research.

2.2. EAE Induction. EAE induction was based on a published protocol [24]. Murine myelin oligodendrocyte glycoprotein (MOG) 35-55 peptide (MOG35-55, MEVGWYRSPFSRVVHLYRNGK) was synthesized with $>99 \%$ purity (SciLight Biotechnology, China). C57BL/6 mice were injected with $200 \mu \mathrm{g}$ MOG35-55 emulsified in $100 \mu \mathrm{g}$ of complete Freund's adjuvant (CFA, Sigma) and an additional $400 \mu \mathrm{g}$ Mycobacterium tuberculosis H37RA (BD Biosciences) by subcutaneous injection into the flanks. These mice were also given $400 \mathrm{ng}$ pertussis toxin (List Biological Laboratories) in $100 \mu \mathrm{l}$ phosphate buffered saline (PBS) on the same day and 2 days later by intraperitoneal injection. In addition, MOG35-55 was administered again 1 week later. Clinical scores were recorded daily for 42 days postimmunization. Neurological function was scored on a $0-5$ scale: 0 , no signs of disease; 1 , partial loss of tail tonicity; 2, tail paralysis; 3 , ataxia and/or paresis of hind limbs; 4 , complete paralysis of hind limbs; and 5, moribund or death [24]. A cumulative clinical score was the sum of all neurological function scores from onset to day 42. Day of onset was when an animal first exhibited neurological signs of disease.

2.3. Fecal Microbiota Transplantation (FMT). C57BL/6 mice (3/cage) were placed in empty autoclaved cages (no bedding) and allowed normal bowel movement. At least twelve fecal pellets were collected from each cage using sterile filter paper, promptly placed in $3 \mathrm{ml}$ sterile $\mathrm{PBS}$, homogenized for $2 \mathrm{~min}$ with a glass pestle and spun at $800 \mathrm{rpm}$ for $3 \mathrm{~min}$ before collecting the supernatant for transplantation. Immunized mice were randomly divided into two groups: one group (FMT group) was given $200 \mu \mathrm{l}$ per mouse fresh fecal supernatant via oral gavage daily for 42 consecutive days postimmunization, whereas for the other group (EAE group), fecal supernatant was replaced with sterile saline.

2.4. Sample Collection and Microbiota Analysis. Fresh feces were collected and immediately stored at $-80^{\circ} \mathrm{C}$. Fecal microbiota DNA was recovered with the PowerFecal DNA Kit (Qiagen). The V3-V4 region of 16S rRNA gene was amplified by using a pair of primers: 338F, $5^{\prime}$-ACTCCTACGGGAGG CAGCAG- ${ }^{\prime}$, and 806R, $5^{\prime}$-GGACTACHVGGGTWTCTA AT- $3^{\prime}$. PCR amplification products were sequenced by paired-end sequencing (Majorbio, China).

2.5. Tissue Preparation. At day 42 postimmunization, mice were perfused transcardially with ice-cold saline under terminal anesthesia. Mice used for immunostaining were further perfused with $4 \%$ paraformaldehyde. Dissected brain and thoracic spinal cord tissues were fixed in $4 \%$ paraformaldehyde for $12-24 \mathrm{~h}$ and then immersed in $20 \%$ and $30 \%$ sucrose each for 1 day. The tissues were embedded in Tissue-Tek OCT (Sakura), frozen, and cut into $25 \mu \mathrm{m}$ thick spinal cord cross sections and brain coronal sections. For evaluation of BBB leakage, 4\% Evans blue (Sigma) in PBS was injected into the tail vein of mice $(3 \mu \mathrm{l} / \mathrm{g})$ under anesthesia two hours before perfusion.

2.6. Immunofluorescence Staining. To detect Claudin 5 expression, thoracic spinal cord sections were sealed with blocking buffer (10\% sheep serum albumin and $0.3 \%$ Triton $\mathrm{X}-100$ in PBS) for $30 \mathrm{~min}$ at $37^{\circ} \mathrm{C}$, followed by incubation with anti-Claudin 5 antibody $(1: 200$, ab15106, Abcam, USA) at $4^{\circ} \mathrm{C}$ overnight. For other examinations by immunostaining, brain sections were sealed with anti-Ibal $(1: 100$, ab153696, Abcam, USA), anti-GFAP (1:200, Cat.\#12389, CST, USA), or anti-MBP $(1: 100$, Cat.\#13344, CST, USA) together with anti-NF-L antibody $(1: 400$, Cat.\#AB9568, Millipore, USA) at $4^{\circ} \mathrm{C}$ for $48 \mathrm{~h}$. Alexa Fluor ${ }^{\circledR} 488$ conjugated goat anti-rabbit secondary antibody $(1: 800$, ab150077, Abcam, USA) was then added on its own or together with Alexa Fluor ${ }^{\circledR}$ 555-conjugated goat anti-mouse antibody ( $1: 800$, ab150118, Abcam, USA) for incubation at $37^{\circ} \mathrm{C}$ for $1 \mathrm{~h}$. Nuclei were counterstained with Hoechst 33342 (Cat.\#C0030, Solarbio, Beijing, China) for $10 \mathrm{~min}$. Photomicrographs were taken with a confocal microscope (Leica TCS SP5 II).

2.7. Transmission Electron Microscopy. Thoracic spinal cord tissues were postfixed in osmium tetroxide and processed for transmission electron microscopy. Electron micrographs were taken with a Jem-1400 transmission electron microscope.

2.8. Western Blotting. The protein was extracted from brain tissues using an Ambion PARIS kit (Solarbio, Beijing, China) with addition of phosphatase and protease inhibitor cocktails (Roche). The Pierce BCA Protein Assay Kit (Cat.\#23227, 
Thermo Fisher) was used to detect protein concentrations. The proteins were separated by SDS-PAGE electrophoresis, and a blotted membrane was incubated with blocking buffer and then with anti-Claudin 5 (1:500, ab15106, Abcam, USA), anti-Ibal (1:1000, ab153696, Abcam, USA), antiGFAP (1:1000, Cat.\#12389, CST, USA), anti-MBP (1:1000, Cat.\#13344, CST, USA), or anti-NF-L (1:1000, Cat.\#AB9568, Millipore, USA) primary antibody at $4^{\circ} \mathrm{C}$ overnight before addition of horseradish peroxidase- (HRP-) linked goat antirabbit ( $1: 1000$, Cat.\#7074, CST, USA) or goat anti-mouse secondary antibody ( $1: 25000$, Cat.E030110-01, EarthOx, USA). Detection of $\beta$-tubulin was performed on stripped membranes with anti- $\beta$-tubulin $(1: 1000$, ab179513, Abcam, USA) primary antibody to control for protein loading. Protein signal was visualized with the LumiGLO chemiluminescent substrate (Cat.\#7003S, CST, USA), and target protein expression was normalized as fold change relative to $\beta$-tubulin expression using Photoshop for quantitative analysis.

2.9. Statistical Analysis. Statistical analysis was performed by the Mann-Whitney $U$ test, followed by a linear discriminant analysis for phylum level changes. The SPSS 17.0 software was used for statistical analysis including two-way analysis of variance (ANOVA) followed by a Bonferroni test for comparing clinical scores and unpaired $t$-tests for other comparisons with means and $p$ values calculated. Statistical graphs were generated with GraphPad Prism 5. $p<0.05$ was considered statistically significant.

\section{Results}

3.1. FMT Modulates GM in EAE. To examine the effect of FMT on GM structure in EAE, fecal samples were collected from EAE mice $(n=6)$, FMT-treated immunized mice $(n=6)$, and normal controls $(n=5) 42$ days postimmunization for DNA extraction and $16 \mathrm{~S}$ rRNA gene sequencing. Acquired sequencing data were analyzed for assessment of GM diversity. We first evaluated GM $\alpha$-diversity with the Shannon index, which takes account of both the richness and evenness of a microbial community. The Shannon index was significantly increased for GM in EAE mice compared to normal controls $(4.38 \pm 0.15$ vs. $3.89 \pm 0.26, p=0.008113)$, indicating altered GM diversity in EAE; an in-between value was discovered in FMT-treated EAE mice without statistical significance (Figure 1(a)), suggesting that FMT attenuated the increase in the Shannon index caused by the development of EAE. Therefore, these results hint that FMT is conducive to restoring altered intestinal microbiota diversity in EAE. We next evaluated GM $\beta$-diversity, which accounts for the dissimilarity between different microbial communities. Principal coordinates analysis (PCoA) of unweighted UniFrac distances showed clear clustering separation between samples from these three mouse groups on scores plot for principal component 1 (PC1, 28.91\%) and PC2 (13.45\%), illustrating differing GM diversity between these mouse groups (Figure $1(\mathrm{c})$ ). The $\beta$-diversity distance matrix was presented for hierarchical clustering analysis to calculate the phylogenetic evolutionary relationships of each species and the distance between samples; pairwise intergroup
UniFrac distances further quantitatively detected the variation occurring on different lineages among samples. Analysis showed that FMT was closer to the control than the EAE mice on the OTU level (Figure 1(d)).

Compared with EAE mice, intestinal bacterial phyla Bacteroidetes $(p=0.0081)$ were more abundant in normal controls, whereas Firmicutes $(p=0.0081)$, Tenericutes $(p=0.0081)$, and Cyanobacteria $(p=0.0354)$ were less abundant. FMT-treated EAE mice presented changed abundances of Verrucomicrobia $(p=0.0091)$, four intestinal bacterial phyla Bacteroidetes, Firmicutes, Tenericutes, and Cyanobacteria, all of which that shifted towards the levels observed in normal controls (Figure 1(b)) even if it is not statistically significant. Those suggest that FMT can to a certain degree remedy altered GM structure in EAE.

To determine which bacteria was associated with the severity of the neurological function score, we performed a Spearman's correlation of bacterial abundance with EAE scores and cumulative disease scores (Figure 1(e)). Both Lachnoclostridium and Unclassified_f_Lachnospiraceae showed negative correlation in EAE scores and EAE cumulative scores. Five kinds of genus including norank_o_Mollicutes_RF9, [Eubacterium]_ruminantium_group, unclassified_f_Ruminococcaceae, Turicibacter, Ruminococcus_1, and Thalassospirawere positively correlated with EAE scores and EAE cumulative scores. However, uncultured_f_Lachnospiraceae, Helicobacter, Roseburia, and norank_f_Bacteroidales_S24-7_ group were found to be negatively correlated with EAE scores and cumulative scores, respectively. Prevotellaceae_UCG-001, Akkermansia, and Ruminococcaceae_UCG-014 showed positive correlation in EAE score, as well as Alistipes, unclassified_ f_Veillonellaceae, Ruminiclostridium_6, Allobaculum, and norank_f_Clostridiales_vadinBB60_group in cumulative scores. These results indicated that different genus of bacteria contributes differently to EAE neurological function score.

Analysis of the GM profiles of these 3 mouse groups using linear discriminant analysis (LDA) of effect size (LEfSe) identified differentially abundant bacterial taxa (LDA score threshold $>2.0$ ), which reflected the effect of EAE and FMT treatment on the abundances of gut bacterial taxa. EAE caused a marked decrease in the abundances of 13 gut bacterial taxa (g_norank_f_Bacteroidales_S24_7_group, $f$ Bacteroidales_S24_7_group, p_Bacteroidetes, $c$ _Bacteroidia, o_ Bacteroidales, g_Ruminococcaceae_UCG_010,f_Family_XIII, g_Eubacerium_nodatum_group, c_Betaproteobateria, g_Parasutterella, f_Alcaligenaceae, o_Burkholderiales, and $g$ Lachnoclostridium), made up of 1 phylum, 2 classes, 2 orders, 3 families, and 5 genera. FMT treatment for EAE decreased the abundances of 17 bacterial taxa ( $p \_$Fimicutes, $f \_$Ruminococcaceae, g_Alloprevotella, g_Alistipes, g_Ruminococcus_1, g_unclassified_f_Ruminococcaceae, g_Ruminococcaceae_ UCG_014,g_Akkermansia, p_Verrucomicrobia,o_Verrucomicrobiales, f_Verrucomicrobiaceae, c_Verrucomicrobiae, $g$ Eubacterium_ruminantium_group, f_Staphylococcaceae, $g$ Staphylococcus, o_Bacillales, and g_Paraprevotella) consisting of 2 phyla, 1 class, 2 orders, 3 families, and 9 genera (including Akkermansia) and increased the abundances of 34 bacterial taxa ( $f \_$Rikenellaceae, f_Bacteroidaceae, g_Bacteroides, $g$ Prevotella_1, g_Turicibacter, g_Prevotellaceae_UCG_003, $f_{-}$ 


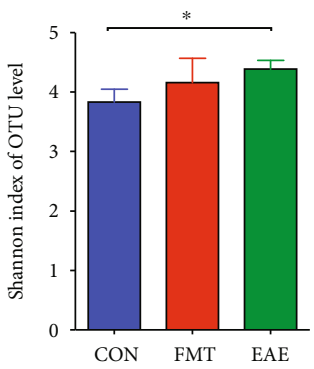

(a)

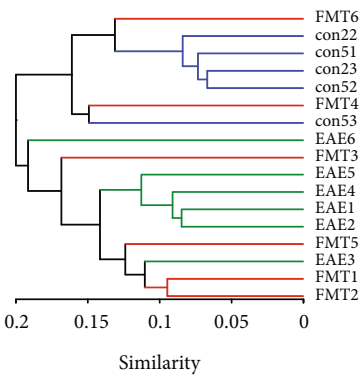

- $\mathrm{fmt}$
二 con
eae

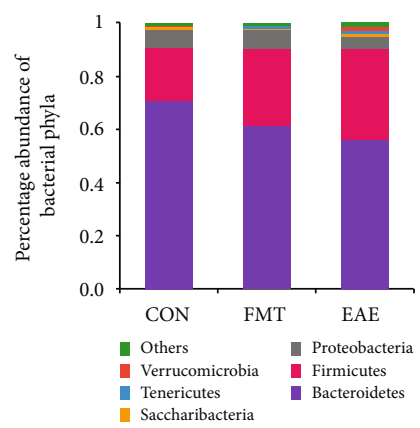

(b)

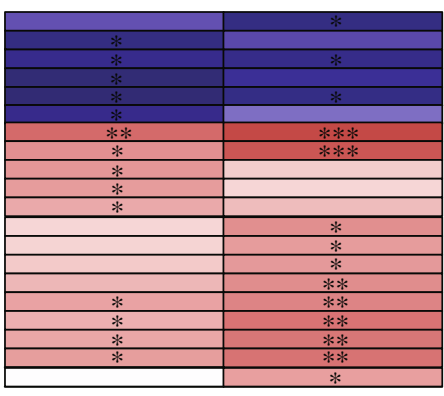

EAE scores

Cumulative scores

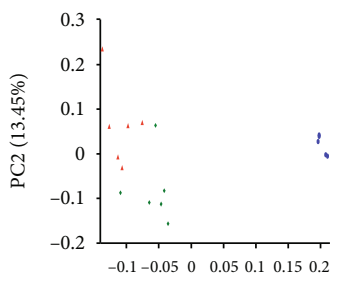

PC1 (28.91\%)

- CON
FMT

(c)

Norank_f_Bacteroidales_S24-7_group Uncultured_f_Lachnospiraceae Lachnoclostridium

Unclassifed f

Roseburia
Norank_o_Mollicutes_RF9

Eubacterium]_ruminantium_group

Akkermansia

Ruminococcaceae_UCG-014

Ulistipes

Ruminiclostridium_6

Allobaculum

Ruminococcus_ 1 -

unclassified__

Turicibacter

Thalassospira
Norank_f_Clostridiales_vadinBB60_group

$-0.5$

(e)

(d)
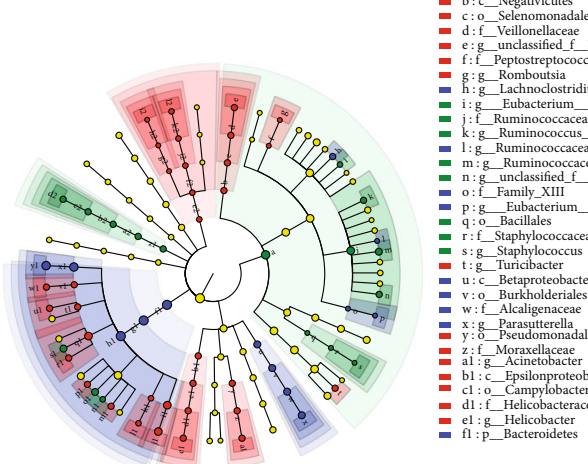

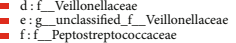

E: gRomboutsia

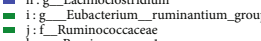

- $\begin{aligned} & \mathrm{k}: \mathrm{g} \_ \text {Ruminococcus_-1 } \\ & 1: \mathrm{g} \text { Ruminococcaceae_UCG_010 }\end{aligned}$

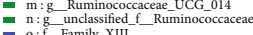

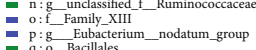

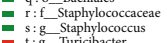

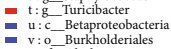

w: f-Alcaligenaceae

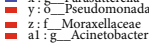

a bi:c_Epsilonproteobacteria

= cl: : C Camplobacter

el : : Helicobact
$\mathrm{f} 1: \mathrm{p}$ Bacteroide

CON

FMT

EAE
- g1:c_Bacteroidia

- h1:o_Bacteroidales

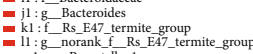

$=0$

- n1:g_Paraprevotella
o o1: : Alloprevotella
- 1 : g_Prevotellacea_UCG_003

- $\mathrm{q1}: \mathrm{f} \_$Rikenellace
$\mathrm{r} 1: \mathrm{g}$-Rikenella
s1: g_Alistipes

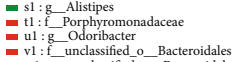

- $\mathrm{v} 1:$ : Cunclassified_o_Bacteroidales

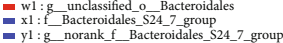

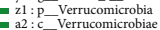

- b2: : Verrucomicrobiales

- $\mathrm{d}^{2}: \mathrm{g}$. Akrkermansia

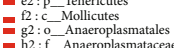

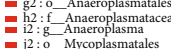

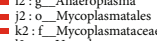

(f)

Figure 1: Continued. 


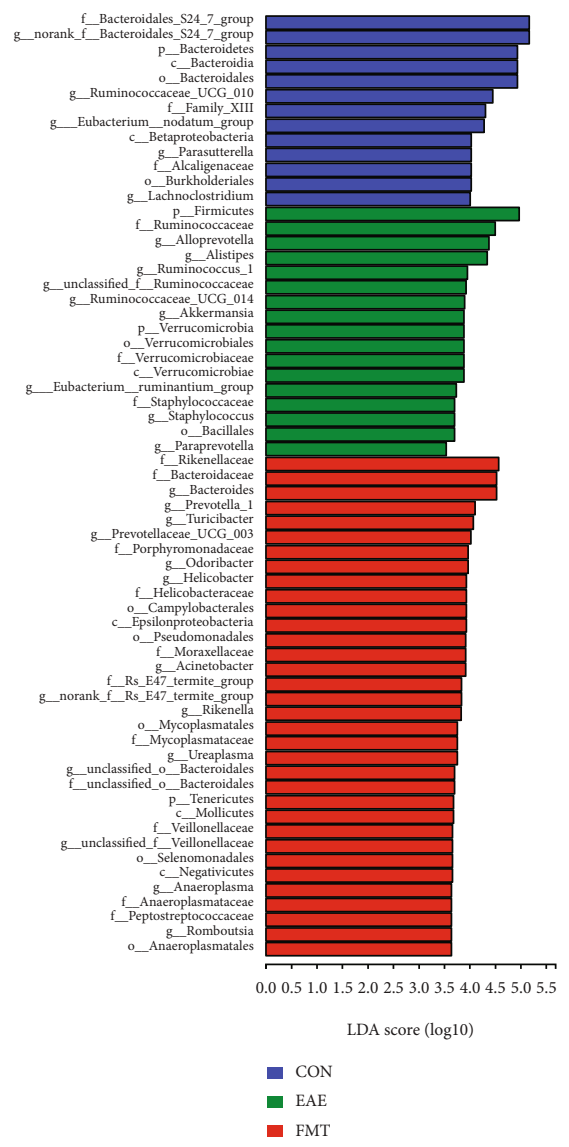

(g)

Figure 1: FMT modulates GM in EAE. (a) Chart of the Shannon index values for evaluation of GM $\alpha$-diversity. The Shannon index for GM was significantly increased in EAE mice $(n=6)$ compared to normal controls (CON, $n=5)$; an in-between value was found in FMT-treated immunized mice (FMT; $n=6$ ) without statistical significance. (b) Chart of relative abundance of gut bacterial taxa (phylum level) performed with the Mann-Whitney $U$ test, corrected by FDR between the two groups. The figure was created by used unfiltered OUT table. (c) PCoA plot (PC1/PC2) of unweighted UniFrac distances illustrating clustering separation between samples from different mouse groups. (d) Hierarchical clustering tree on OTU level (weighted UniFrac). (e) The correlation heatmap chart performed with a Spearman's correlation of bacterial abundance with EAE scores and cumulative scores. The $R$ value is shown in different colors in the figure. The legend on the right is the color interval of different $R$ values $(* 0.01<p<0.05$ and $* * 0.001<p<0.01)$. (f) LEfSe cladogram showing differentially abundant gut bacterial taxa. The diameter of each dot is proportional to its effect size. Each ring (from inside to outside) represents a taxonomic level from kingdom to genus, the cladogram was made on filtered data, and only taxa with greater than $0.1 \%$ relative abundance. (g) LDA scores of abundant gut bacterial taxa (LDA score threshold >2.0). k: kingdom; p: phylum; c: class; o: order; f: family; g: genus.

Porphyromonadaceae, g_Odoribacter, g_Helicobacter, $f_{-}$ Helicobacteraceae, o_Campylobacterales, c_Epsilonproteobacteria, o_Pseudomonadales, $f \_$Moraxellaceae, g_Acinetobacter, $f \_R s \_E 47$ termite_group, g_norank_f_Rs_E47_termite_group, g_Rikenella, o_Mycoplasmatales, $f_{-}$Mycoplasmataceae, $g_{-}$ Ureaplasma, g_unclassified_o_Bacteroidales, f_unclassified_ o_Bacteroidales, $p \_T e n e r i c u t e s, c \_$Mollicutes, $f$ _Veillonellaceae, g_unclassified_f_Veillonellaceae, o_Selenomonadales, c_Negativicutes, g_Anaeroplasma, f_Anaeroplasmataceae, f_Peptostreptococcaceae, g_Romboutsia, and o_Anaeroplasmatales increased in FMT mice) comprising 1 phylum, 3 classes, 5 orders, 11 families, and 14 genera (including Prevotella) in GM (Figures 1(f) and 1(g)). Taken together, these results demonstrate that FMT can modulate GM, thereby to some extent rectifying altered GM composition in EAE.
3.2. FMT Has a Therapeutic Effect on EAE. Study of human patients has revealed distinct GM in MS [25], and research with animal models of MS has discovered that modulating GM with antibiotics and probiotics can decrease EAE clinical severity [26]. To find out the effect of FMT on EAE clinical symptoms, we evaluated clinical scores of immunized mice with versus without FMT treatment. FMT-treated mice $(n=10)$ displayed alleviated clinical symptoms evidenced by significantly reduced clinical scores $(p<0.0001)$ and cumulative disease scores $(p<0.05)$ compared with EAE controls $(n=10)$ through the clinic course of EAE (Figures 2(a) and 2(b)). Furthermore, FMT treatment resulted in a delay in the onset of EAE $(p<0.0001$, Figure 2(c)). Therefore, FMT with fecal matter from normal donors proved effective in slowing down EAE development and relieving EAE symptoms. 


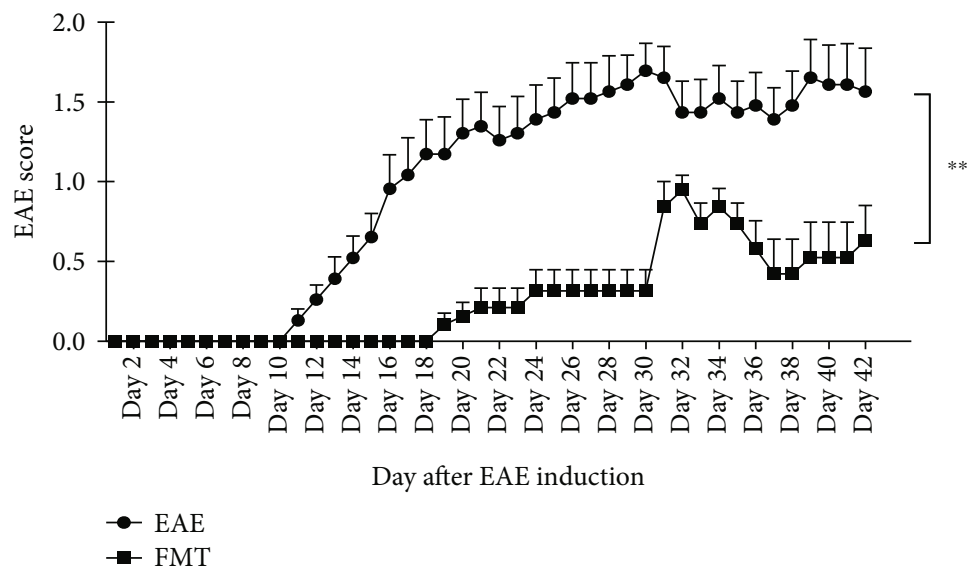

(a)

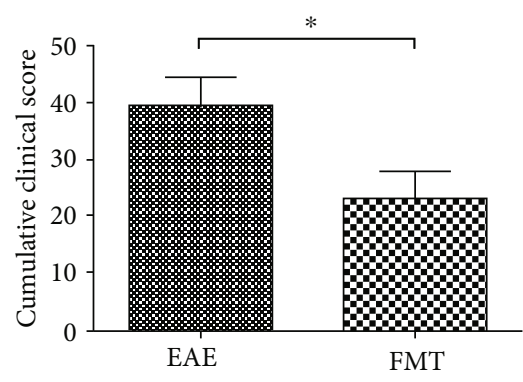

(b)

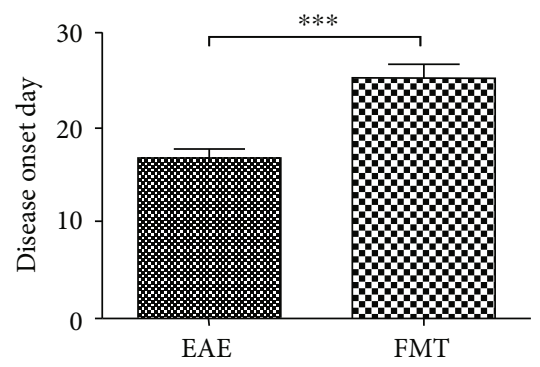

(c)

Figure 2: FMT has a therapeutic effect on EAE. (a) Chart of clinical scores for EAE controls and FMT-treated immunized mice after EAE induction. FMT led to decreased clinical scores through the clinical course of EAE (mean \pm SEM; $n=10 /$ group; $* * p<0.01$ ). (b, c) Charts of cumulative clinical scores (b) and disease onset days (c) after EAE induction indicating reduced clinical severity and delayed disease onset after FMT treatment (mean \pm SEM; $n=10$ /group; $* p<0.05$ and $* * * p<0.001$ ).

3.3. FMT Prevents BBB Leakage in EAE. The BBB is compromised during the development of MS and EAE, allowing immune cells to infiltrate CNS and attack myelin [27]. Because GM is thought able to regulate BBB permeability [28-30], we then investigated whether FMT can help prevent BBB impairment in EAE. By Western blotting, we found that the expression of Claudin 5, a tight junction protein responsible for $\mathrm{BBB}$ barrier function [31], was dramatically increased in brain tissue in FMT-treated immunized mice compared to EAE controls $(n=6)$ (Figures 3(a) and 3(b)), which was further verified by immunostaining of brain sections $(n=3)$ (Figure $3(\mathrm{c}))$. In addition, Evans blue dye staining showed an appreciable reduction in dye presence in brain parenchyma after FMT treatment $(n=1)$ (Figure 3(d)). Together, these results prove that FMT treatment can lead to improved BBB integrity in EAE, preventing $\mathrm{BBB}$ leakage.

3.4. FMT Confers Protection on Myelin and Axons in EAE. To assess the influence of FMT on myelin and axons, we examined the expression of myelin basic protein (MBP), which is expressed in myelin, and neurofilament light chain protein (NF-L), whose release reflects axonal damage, in brain tissue. Significantly increased MBP expression and decreased NF-L expression were detected in brain tissue in FMT-treated EAE mice compared to saline-treated controls by Western blotting (Figures 4(a)-4(c)) and by immunostaining of brain sections as well (Figure 4(d)), indicating an increase in the number of normal myelin sheaths and a decrease in myelin disintegration and axon damage after FMT treatment. Moreover, transmission electron microscopy (TEM) verified lessened demyelination and increased the presence of intact myelin sheaths in the thoracic spinal cord after FMT treatment (Figure 4(e)). All together, these data point to a protective effect conferred by FMT on myelin and axons in EAE.

3.5. FMT Alleviates Microglia and Astrocyte Activation in $E A E$. Microglia and astrocytes are known to contribute to the inflammatory pathology of MS [32], and some studies hint at a connection between their activation and GM composition $[33,34]$. To find out the impact of FMT on microglia and astrocyte activation, we examined the expression of ionizing calcium-binding adaptor molecule 1 (Iba1, immune cells marker, which is not specific for microglia and infiltrating monocyte) and glial fibrillary acidic protein (GFAP, astrocyte marker) with Western blotting and discovered that the expression of both markers was significantly downregulated in FMT-treated EAE mice compared to 


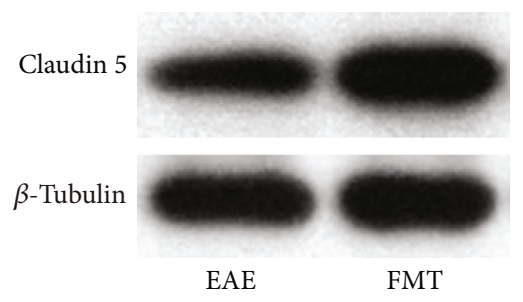

(a)

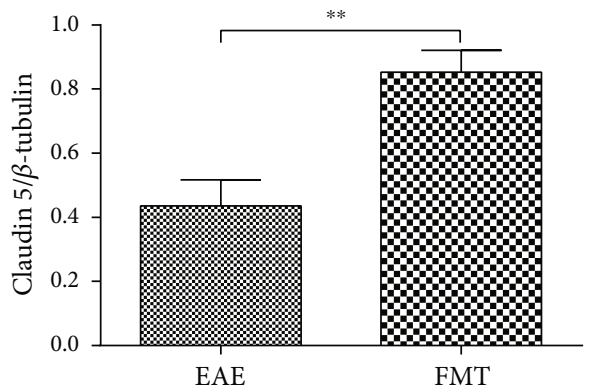

(b)

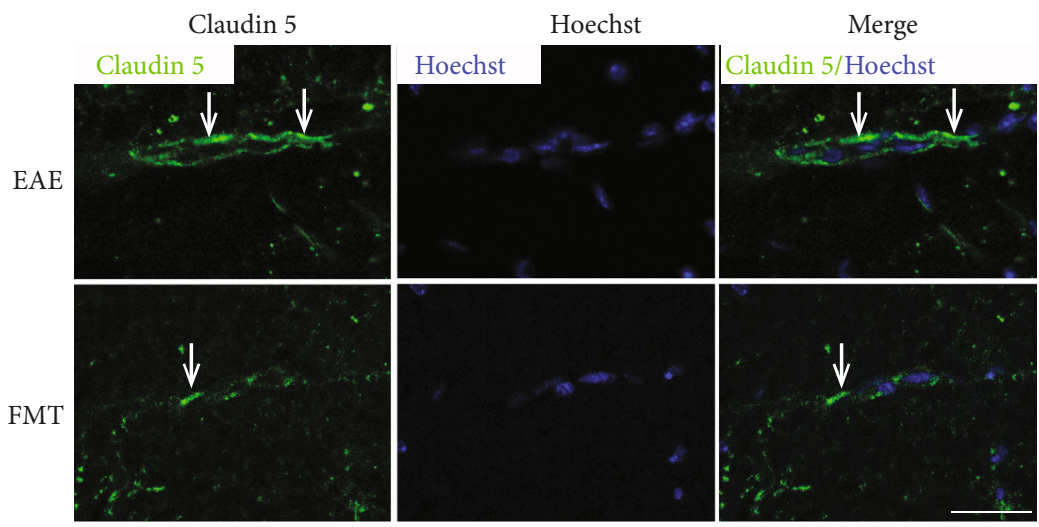

(c)

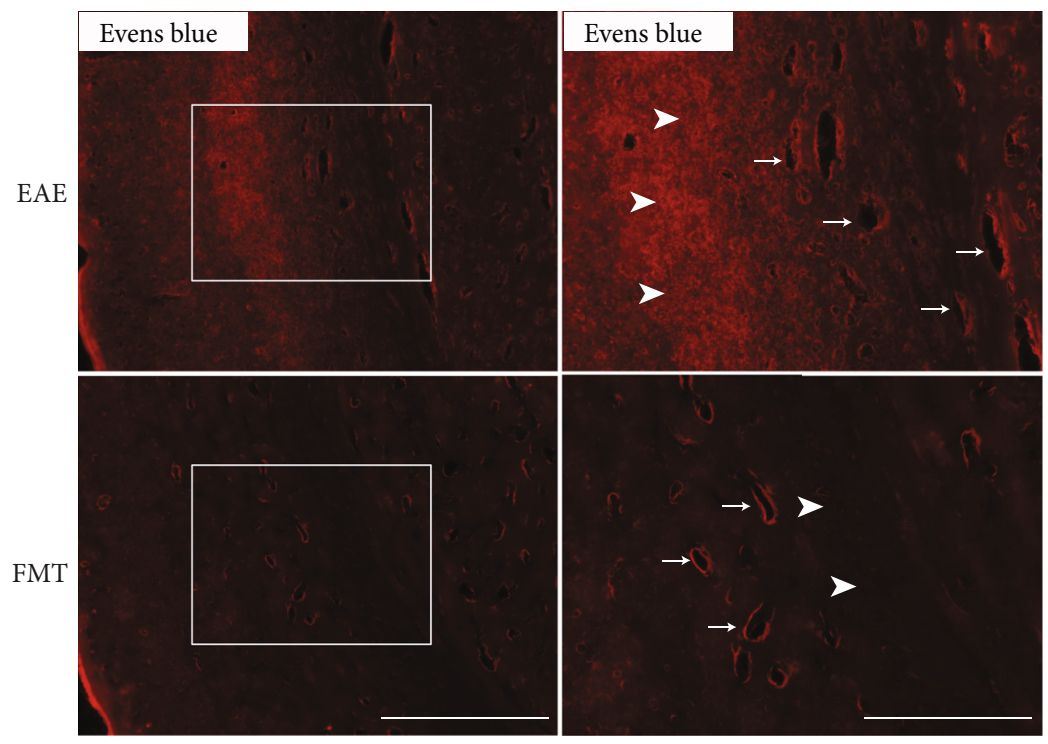

(d)

FIGURE 3: FMT prevents BBB leakage in EAE. (a) Claudin 5 expression in thoracic spinal cord tissues collected from EAE controls and FMTtreated EAE mice revealed by Western blot with $\beta$-tubulin as loading control. (b) Chart of quantified Western blot results showing increased levels of Claudin 5 expression (normalized by $\beta$-tubulin) after FMT treatment (mean \pm SEM; $n=6 /$ group; $* * p<0.01$ ). (c) Representative immunostaining images of Claudin 5 expression (green) in spinal cord sections. The nucleus was stained with Hoechst (blue). Scale bar: $100 \mu \mathrm{m}$. (d) Representative images of Evans blue dye extravasation (red) in the subcortical white matter of brain showing the presence of dye in both blood vessels and brain parenchyma in EAE controls (upper panels) in contrast to appreciably reduced dye presence in brain parenchyma in FMT-treated EAE mice (lower panels). Each right panel shows a high magnification image of the area inside the white box (left). Scale bars: $400 \mu \mathrm{m}$ (left) and $200 \mu \mathrm{m}$ (right). Representative brain parenchyma and blood vessels are indicated by arrowheads and arrows, respectively.

saline-treated controls (Figures 5(a)-5(c)). Furthermore, decreased numbers of microglia and astrocytes were observed by immunostaining of brain sections after FMT treatment (Figures 5(d) and 5(e)). Taken together, these data imply subdued glial inflammatory response after FMT treatment in EAE. 


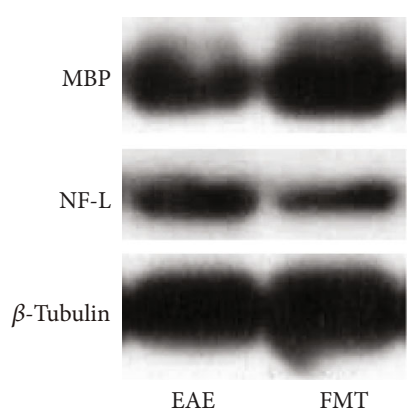

(a)

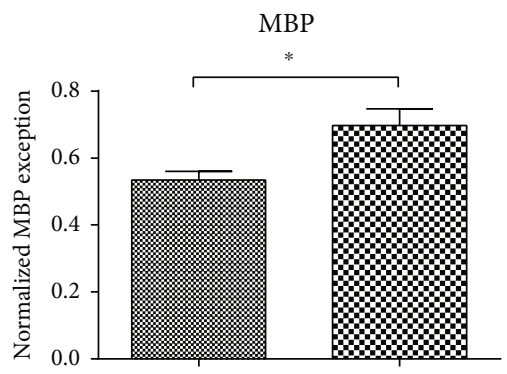

FMT

(b)

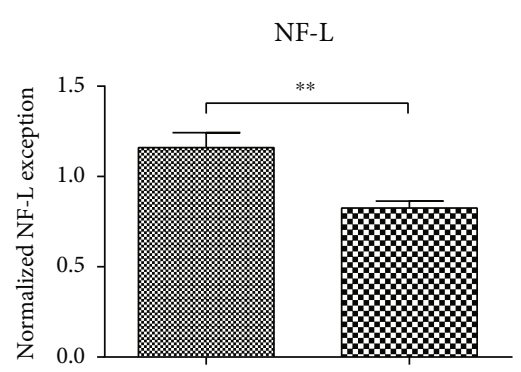

EAE
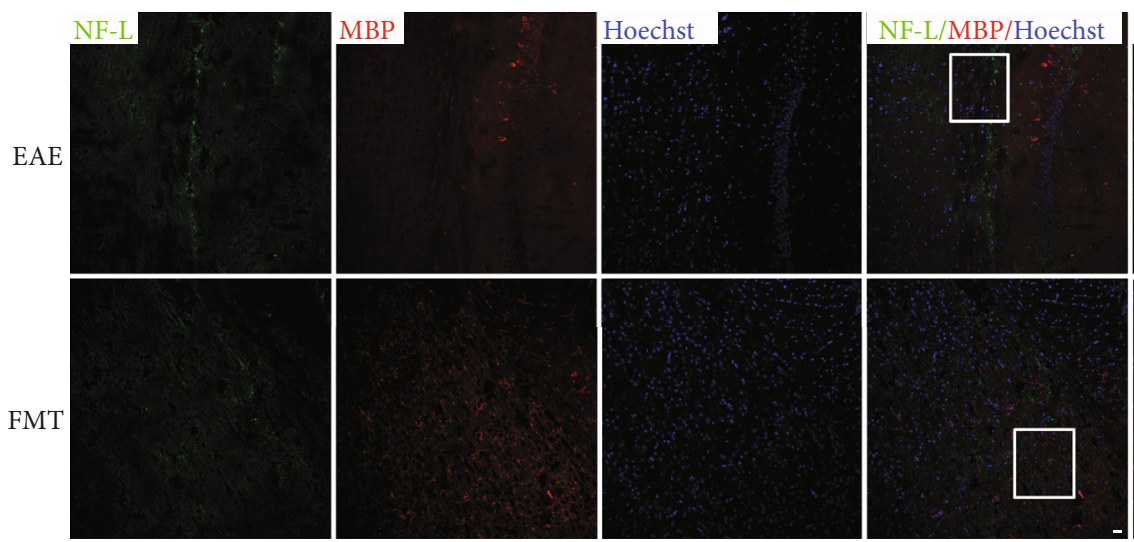

(c)

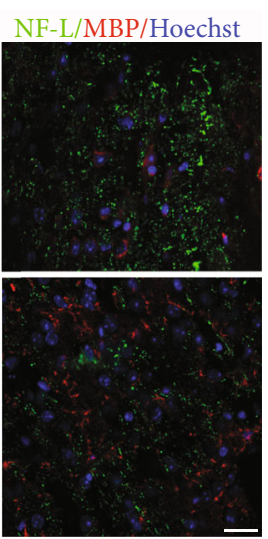

(d)

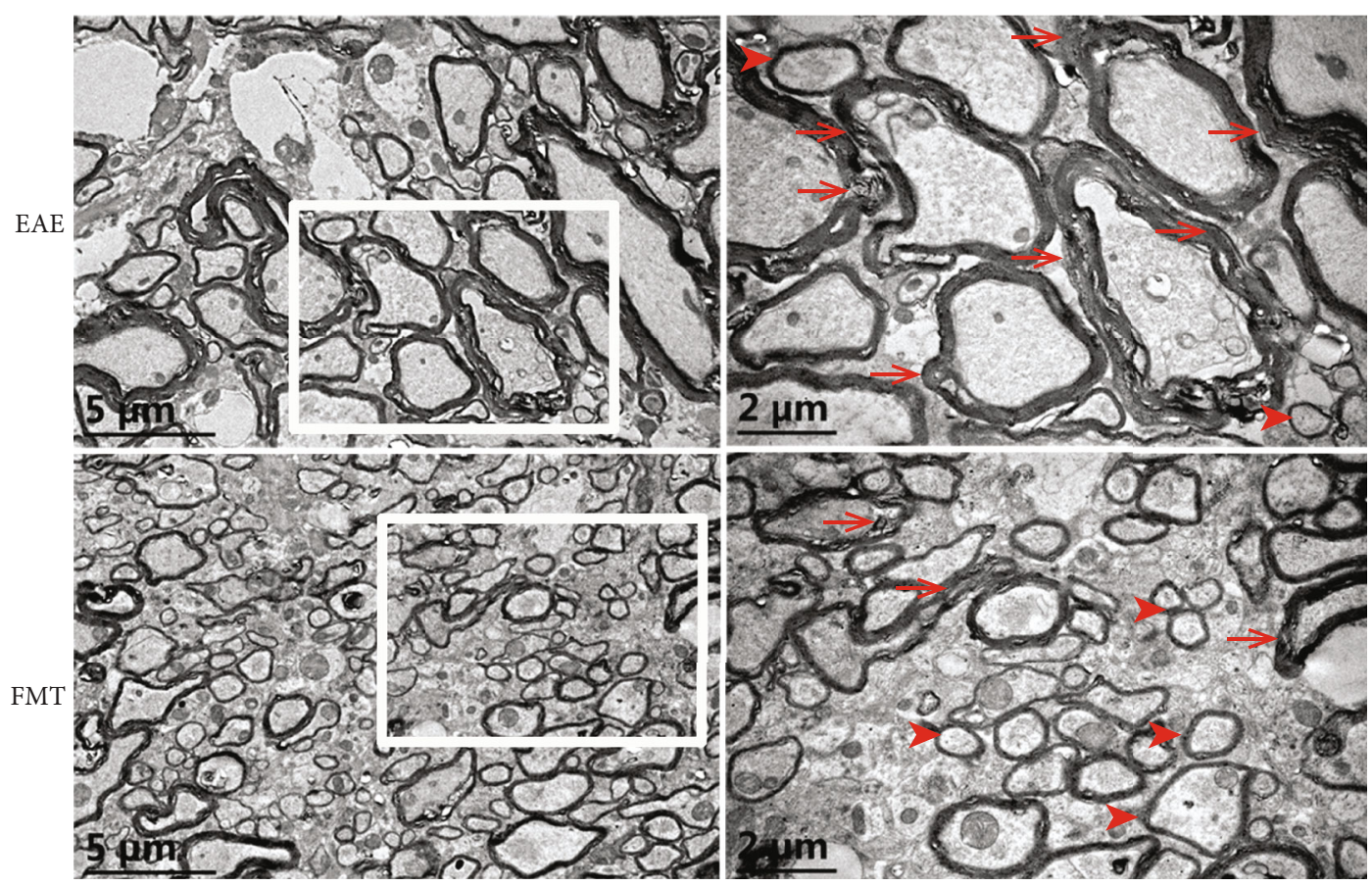

(e)

FIGURE 4: FMT confers protection on myelin and axons in EAE. (a) MBP and NF-L protein expression in the brain of EAE controls and FMTtreated EAE mice shown by Western blot with $\beta$-tubulin as loading control. (b, c) Charts of quantified Western blot results showing increased levels of MBP (b) and decreased levels of NF-L (c) expression (normalized by $\beta$-tubulin) after FMT treatment (mean \pm SEM; $n=6 /$ group; $* p<0.05$ and $* * p<0.01$ ). (d) Immunofluorescence imaging of MBP (green) and NF-L (red) expression in the corpus callosum of the mouse brain. Nuclear staining was by Hoechst (blue). Each rightmost panel shows a high magnification image of the area inside the white box. Scale bar: $25 \mu \mathrm{m}$. (e) Transmission electron microscopy (TEM) imaging of myelin sheaths in the thoracic spinal cord. Each high magnification image (right) shows the area enclosed by the white box (left). Representative normal and damaged myelin sheaths are indicated by arrowheads and arrows, respectively. 


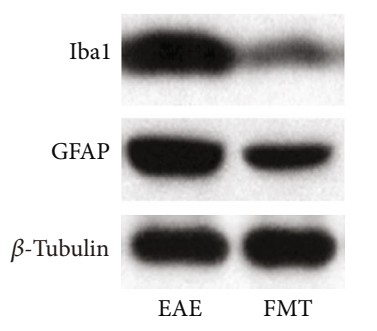

(a)

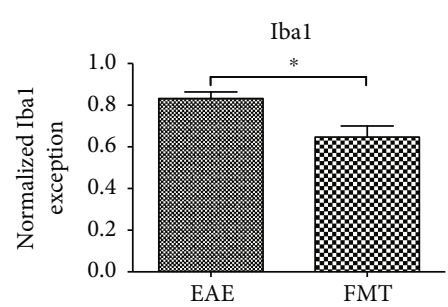

(b)

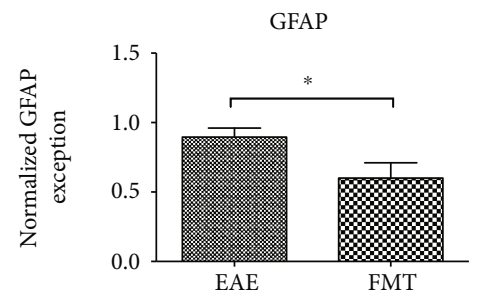

(c)
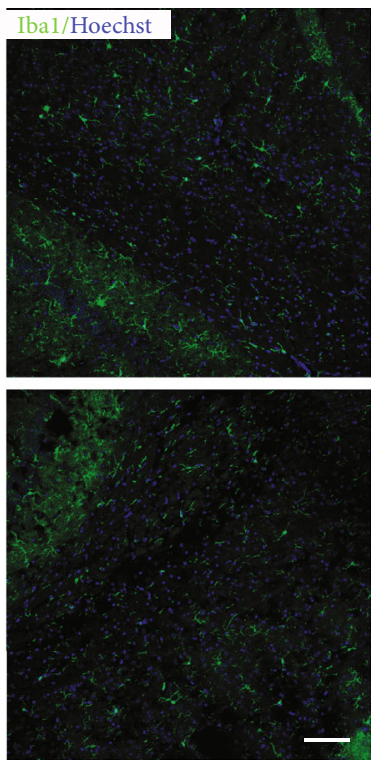

(d)
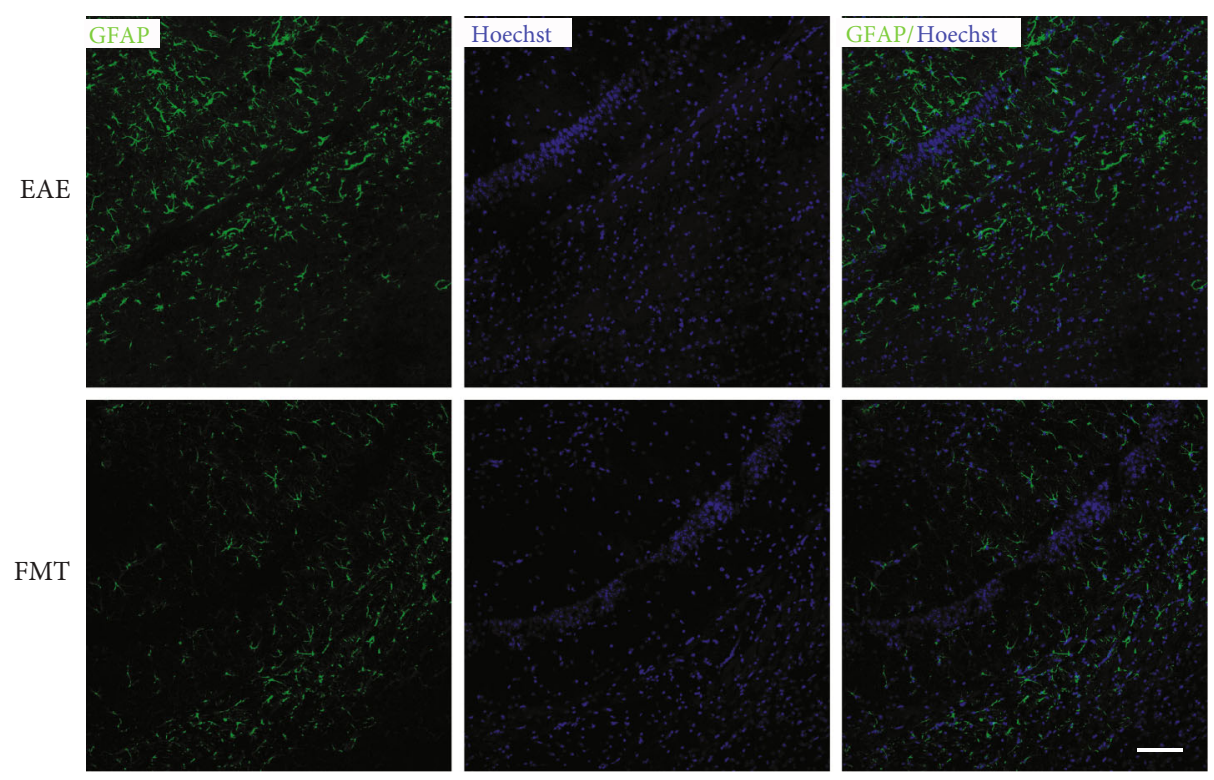

(e)

FIGURE 5: FMT alleviates immune cells and astrocyte activation in EAE. (a). Expression of Ibal and GFAP protein in the brain of EAE controls and FMT-treated EAE mice shown by Western blot with $\beta$-tubulin as loading control. (b, c). Charts of quantified Western blot results indicating reduced levels of Ibal(b) and GFAP (c) expression (normalized by $\beta$-tubulin) after FMT treatment (mean \pm SEM; $n=6$ /group; $* p<0.05$ ). (d, e) Immunofluorescence imaging of cells expressing Iba1 (green; d) and GFAP (green; e) in brain sections with the nucleus stained by Hoechst (blue). Scale bar: $100 \mu \mathrm{m}$. 


\section{Discussion}

An increasing body of evidence reveals GM dysbiosis in MS $[16,25,35,36]$, and rebuilding GM has been proposed as an innovative approach to MS treatment. FMT appears to be the most direct way to reconstruct GM $[37,38]$ and is in fact an ancient treatment dating back to 1700 years ago [39]. However, whether GM can be restored back to normal by FMT remains unclear. Our data reveal a tendency for GM structure to change towards normal after FMT treatment in EAE with beneficial consequences (Figure 1).

We also found that FMT treatment for EAE markedly reduced the abundance of Akkermansia genus (in phylum Verrucomicrobia) and elevated the abundance of Prevotella genus (in phylum Bacteroidetes) in GM (Figures 1(b), 1(f), and $1(\mathrm{~g})$ ), which recalls the findings of decreased gut Akkermansia after probiotic intervention [40, 41], and increased gut Prevotella after disease-modifying treatment [16] and intermittent fasting in MS patients [42]. Bacteroidetes is one of the most abundant bacterial phyla inhabiting human gut, and the Prevotella genus is a dominant member of this phylum. Bacteroidetes ferments dietary fibers to produce short chain fatty acids (SCFAs), which take part in various physiological processes, affecting host health [43-45]. SCFAs have been found to protect the BBB from oxidative stress via nuclear factor, erythroid 2-like 2 (NFE2L2) signaling [46] and exert anti-inflammatory and neuroprotective functions [47-49]. Other favorable effects of SCFAs include attenuating myelin injury by increasing brain acetyl-CoA metabolism [50] and relieving clinical symptoms in EAE mice $[49,50]$. A zwitterionic capsular polysaccharide A (PSA) produced by Bacteroides fragilis suppresses neuroinflammation by regulating migratory capacity of $\mathrm{CD}_{39}{ }^{+} \mathrm{CD} 4 \mathrm{~T}$ cell subsets, thus ameliorates EAE [51]. Bacteroides fragilis PSA+ regulated CNS demyelination by the induction of highly potent IL-10-producing Treg cells in EAE [52]. In addition, increased gut Akkermansia is associated with MS [16, 40], and probiotic treatment for MS patients resulted in decreased Akkermansia in GM accompanied by an anti-inflammatory peripheral response [40]. Moreover, increase of gut Akkermansia has been implicated in Parkinson's disease [53, 54] and advanced dementia [55]; these results showed a negative role for intestinal Akkermansia in CNS disorders. While Akkermansia is consistently elevated in MS subjects, however, it may be a compensatory change for a study has shown that transferring Akkermansia to mice at EAE onset can ameliorate disease [56].

So far, FMT has been tested in treating a variety of conditions including Clostridium difficile infection [42, 57], active ulcerative colitis [58], high-fat diet-induced steatohepatitis [59], metabolic syndrome [60], and CNS diseases such as epilepsy [21] and autism spectrum disorder [61]. Notably, applying FMT to several MS patients [62] achieved promising improvement of clinical outcome and FMT treatment for EAE mice with fecal matter from immunized mice on intermittent fasting ameliorated EAE clinical course [42]. In this study, FMT had a therapeutic effect on EAE, reducing clinical severity and delaying the onset of disease (Figure 2). Hence, our data add more weight to the idea of using FMT as a GM-based treatment for MS.

Currently, how FMT exerts influence on MS remains unclear. Evidence from the EAE model of MS suggests a critical role for GM and its metabolites in the mechanisms behind neuroinflammation and demyelination $[17,18]$. Our attempt to rectify altered GM in EAE by FMT led to alleviated neuroinflammation and reduced BBB leakage and damage to myelin and axons (Figures 3-5). These results lend support to GM's involvement in the pathogenesis of EAE and echo previous observations of GM regulating $\mathrm{BBB}$ integrity [28], myelination [63], and microglia activation $[29,64]$. It is worth noting that two earlier studies experimented with applying FMT to EAE animals, but the fecal matter used for transplantation came from donor animals under specific experimental conditions [42, 65]. FMT from immunized donor mice on intermittent fasting resulted in reduced inflammation and demyelination in EAE recipient mice, proving that GM was part of the reason for fasting to take effect in treating EAE [42]. FMT from Albino Oxford (AO) donor rats, which are highly resistant to EAE induction, to EAE-prone Dark Agouti (DA) recipient rats from birth led to ameliorated EAE symptoms and decreased production of interleukin- (IL-) 17, a proinflammatory cytokine, in the spinal cord [65], hinting that GM can confer resistance to EAE.

Our study has limitations. Firstly, we started FMT treatment before the onset of neurological signs, which is a disadvantage from a translational point of view as treatment for MS is sought after the onset of disease. We also did not analyze the ingredients of the fecal matter used for transplantation.

\section{Conclusions}

In summary, our data demonstrate beneficial effects of FMT in the EAE mouse model of MS, including improved GM composition, ameliorated clinical course, subdued glial inflammatory response, and protection conferred on the $\mathrm{BBB}$, myelin, and axons. Our findings suggest a causal linkage between GM and MS pathogenesis, and therefore, GM has the potential to be a new target of innovative therapies for MS. Further work is needed to unravel the mechanisms underlying the impact of FMT on gut-brain axis and formulate an ideal microbial recipe for MS treatment.

\section{Data Availability}

The raw sequences were deposited in the NCBI Sequence Read Archive (SRA) with the accession number SRP201403.

\section{Conflicts of Interest}

The authors declare that there is no conflict of interests.

\section{Authors' Contributions}

KLL, SCW, LH, XJY, YRM, CMJ, and XPP conducted the experiments. ZL, BZ, and HLL designed the experiments. Data analysis was carried out by XXC, ZKH, and HHZ. The 
manuscript was written by ZL and GDM with editorial assistance of HLL. Kanglan Li, Shouchao Wei, Li Hu, and Xiaojian Yin contributed equally to this work.

\section{Acknowledgments}

This work was supported by funding from the Natural Science Foundation of China (NSFC, No. 81400986), the Natural Science Foundation of Guangdong Province (No. 2020A1515011284), the Innovation Project of Ordinary Colleges and Universities in Guangdong (No. 2019KTSCX045), the Science and Technology Planning Project of Zhanjiang (No. 2018A01021), the Medical Scientific Research Foundation of Guangdong Province (No. A2020282), the Traditional Chinese Medicine Bureau of Guangdong Province (No. 20182074), GMU (No. M2016037), and the Affiliated Hospital of GMU (BJ201515, BJ201612, and LCYJ2018A003).

\section{References}

[1] T. Kalincik, "Multiple sclerosis relapses: epidemiology, outcomes and Management. A Systematic Review," Neuroepidemiology, vol. 44, no. 4, pp. 199-214, 2015.

[2] The International Multiple Sclerosis Genetics Consortium \& The Wellcome Trust Case Control Consortium 2, "Genetic risk and a primary role for cell-mediated immune mechanisms in multiple sclerosis," Nature, vol. 476, no. 7359, pp. 214-219, 2011.

[3] C. Cotsapas and D. A. Hafler, "Immune-mediated disease genetics: the shared basis of pathogenesis," Trends in Immunology, vol. 34, no. 1, pp. 22-26, 2013.

[4] A. Nylander and D. A. Hafler, "Multiple sclerosis," The Journal of Clinical Investigation, vol. 122, no. 4, pp. 1180-1188, 2012.

[5] M. Adamczyk-Sowa, A. Medrek, P. Madej, W. Michlicka, and P. Dobrakowski, "Does the gut microbiota influence immunity and inflammation in multiple sclerosis pathophysiology?," Journal of Immunology Research, vol. 2017, 14 pages, 2017.

[6] G. Sharon, N. J. Cruz, D. W. Kang et al., "Human gut microbiota from autism spectrum disorder promote behavioral symptoms in mice," Cell, vol. 177, no. 6, pp. 1600-1618.e17, 2019, e1617.

[7] E. Cekanaviciute, B. B. Yoo, T. F. Runia et al., "Gut bacteria from multiple sclerosis patients modulate human $\mathrm{T}$ cells and exacerbate symptoms in mouse models," Proceedings of the National Academy of Sciences of the United States of America, vol. 114, no. 40, pp. 10713-10718, 2017.

[8] K. Berer, L. A. Gerdes, E. Cekanaviciute et al., "Gut microbiota from multiple sclerosis patients enables spontaneous autoimmune encephalomyelitis in mice," Proceedings of the National Academy of Sciences of the United States of America, vol. 114, no. 40, pp. 10719-10724, 2017.

[9] L. Wrzosek, D. Ciocan, P. Borentain et al., "Transplantation of human microbiota into conventional mice durably reshapes the gut microbiota," Scientific Reports, vol. 8, no. 1, p. 6854, 2018.

[10] J. Ochoa-Repáraz, D. W. Mielcarz, L. E. Ditrio et al., "Role of gut commensal microflora in the development of experimental autoimmune encephalomyelitis," Journal of Immunology, vol. 183, no. 10, pp. 6041-6050, 2009.
[11] S. Stanisavljević, J. Lukić, M. Momčilović et al., "Gut-associated lymphoid tissue, gut microbes and susceptibility to experimental autoimmune encephalomyelitis," Beneficial Microbes, vol. 7, no. 3, pp. 363-373, 2016.

[12] Y. Belkaid and T. W. Hand, "Role of the microbiota in immunity and inflammation," Cell, vol. 157, no. 1, pp. 121-141, 2014.

[13] N. Kamada, S. U. Seo, G. Y. Chen, and G. Nunez, "Role of the gut microbiota in immunity and inflammatory disease," Nature Reviews. Immunology, vol. 13, no. 5, pp. 321-335, 2013.

[14] R. Horai, C. R. Zárate-Bladés, P. Dillenburg-Pilla et al., "Microbiota-dependent activation of an autoreactive $\mathrm{T}$ cell receptor provokes autoimmunity in an immunologically privileged site," Immunity, vol. 43, no. 2, pp. 343-353, 2015.

[15] J. T. Van Praet, E. Donovan, I. Vanassche et al., "Commensal microbiota influence systemic autoimmune responses," The EMBO Journal, vol. 34, no. 4, pp. 466-474, 2014.

[16] S. Jangi, R. Gandhi, L. M. Cox et al., "Alterations of the human gut microbiome in multiple sclerosis," Nature Communications, vol. 7, no. 1, p. 12015, 2016.

[17] K. Berer, M. Mues, M. Koutrolos et al., "Commensal microbiota and myelin autoantigen cooperate to trigger autoimmune demyelination," Nature, vol. 479, no. 7374, pp. 538-541, 2011.

[18] Y. K. Lee, J. S. Menezes, Y. Umesaki, and S. K. Mazmanian, "Proinflammatory T-cell responses to gut microbiota promote experimental autoimmune encephalomyelitis," Proceedings of the National Academy of Sciences of the United States of America, vol. 108, Supplement_1, pp. 4615-4622, 2011.

[19] Y. T. Li, H. F. Cai, Z. H. Wang, J. Xu, and J. Y. Fang, "Systematic review with meta-analysis: long-term outcomes of faecal microbiota transplantation for Clostridium difficile infection," Alimentary Pharmacology \& Therapeutics, vol. 43, no. 4, pp. 445-457, 2016.

[20] S. Paramsothy, R. Paramsothy, D. T. Rubin et al., "Faecal microbiota transplantation for inflammatory bowel disease: a systematic review and meta-analysis," Journal of Crohn's \& Colitis, vol. 11, no. 10, pp. 1180-1199, 2017.

[21] Z. He, B. T. Cui, T. Zhang et al., "Fecal microbiota transplantation cured epilepsy in a case with Crohn's disease: the first report," World Journal of Gastroenterology, vol. 23, no. 19, pp. 3565-3568, 2017.

[22] M. Q. Xu, H. L. Cao, W. Q. Wang et al., "Fecal microbiota transplantation broadening its application beyond intestinal disorders," World Journal of Gastroenterology, vol. 21, no. 1, pp. 102-111, 2015.

[23] T. J. Borody, L. J. Brandt, and S. Paramsothy, "Therapeutic faecal microbiota transplantation: current status and future developments," Current Opinion in Gastroenterology, vol. 30, no. 1, pp. 97-105, 2014.

[24] X. Zheng, X. Hu, G. Zhou et al., "Soluble egg antigen from Schistosoma japonicum modulates the progression of chronic progressive experimental autoimmune encephalomyelitis via Th2-shift response," Journal of Neuroimmunology, vol. 194, no. 1-2, pp. 107-114, 2008.

[25] J. Chen, N. Chia, K. R. Kalari et al., "Multiple sclerosis patients have a distinct gut microbiota compared to healthy controls," Scientific Reports, vol. 6, no. 1, p. 28484, 2016.

[26] W. J. van den Hoogen, J. D. Laman, and B. A. 't Hart, "Modulation of multiple sclerosis and its animal model experimental 
autoimmune encephalomyelitis by food and gut microbiota," Frontiers in Immunology, vol. 8, p. 1081, 2017.

[27] S. Floris, E. L. A. Blezer, G. Schreibelt et al., "Blood-brain barrier permeability and monocyte infiltration in experimental allergic encephalomyelitis," Brain, vol. 127, no. 3, pp. 616$627,2004$.

[28] V. Braniste, M. Al-Asmakh, C. Kowal et al., "The gut microbiota influences blood-brain barrier permeability in mice," Science Translational Medicine, vol. 6, no. 263, p. 263ra158, 2014.

[29] D. Erny, A. L. H. de Angelis, D. Jaitin et al., "Host microbiota constantly control maturation and function of microglia in the CNS," Nature Neuroscience, vol. 18, no. 7, pp. 965-977, 2015.

[30] A. F. Logsdon, M. A. Erickson, E. M. Rhea, T. S. Salameh, and W. A. Banks, "Gut reactions: how the blood-brain barrier connects the microbiome and the brain," Experimental Biology and Medicine (Maywood, N.J.), vol. 243, no. 2, pp. 159-165, 2018.

[31] J. Lv, W. Hu, Z. Yang et al., "Focusing on claudin-5: a promising candidate in the regulation of BBB to treat ischemic stroke," Progress in Neurobiology, vol. 161, pp. 79-96, 2018.

[32] J. Correale and M. F. Farez, "The role of astrocytes in multiple sclerosis progression," Frontiers in Neurology, vol. 6, p. 180, 2015.

[33] H.-J. Lee, Y.-H. Hwang, and D.-H. Kim, "Lactobacillus plantarumC29-Fermented soybean (DW2009) alleviates memory impairment in 5XFAD transgenic mice by regulating microglia activation and gut microbiota composition," Molecular Nutrition \& Food Research, vol. 62, no. 20, article e1800359, 2018.

[34] M. F. Sun, Y. L. Zhu, Z. L. Zhou et al., "Neuroprotective effects of fecal microbiota transplantation on MPTP-induced Parkinson's disease mice: gut microbiota, glial reaction and TLR4/TNF-alpha signaling pathway," Brain, Behavior, and Immunity, vol. 70, pp. 48-60, 2018.

[35] S. Miyake, S. Kim, W. Suda et al., "Dysbiosis in the gut microbiota of patients with multiple sclerosis, with a striking depletion of species belonging to clostridia XIVa and IV clusters," PLoS One, vol. 10, no. 9, article e0137429, 2015.

[36] H. Tremlett, D. W. Fadrosh, A. A. Faruqi et al., "Gut microbiota in early pediatric multiple sclerosis: a case-control study," European Journal of Neurology, vol. 23, no. 8, pp. 1308-1321, 2016.

[37] J. S. Bajaj, G. Kakiyama, T. Savidge et al., "Antibiotic-associated disruption of microbiota composition and function in cirrhosis is restored by fecal transplant," Hepatology, vol. 68, no. 4, pp. 1549-1558, 2018.

[38] B. H. Mullish, J. A. K. McDonald, M. R. Thursz, and J. R. Marchesi, "Antibiotic-associated disruption of microbiota composition and function in cirrhosis is restored by fecal transplant," Hepatology, vol. 68, no. 3, p. 1205, 2018.

[39] F. Zhang, W. Luo, Y. Shi, Z. Fan, and G. Ji, "Should we standardize the 1,700-year-old fecal microbiota transplantation?," American Journal of Gastroenterology, vol. 107, no. 11, pp. 1755-1756, 2012.

[40] S. K. Tankou, K. Regev, B. C. Healy et al., "A probiotic modulates the microbiome and immunity in multiple sclerosis," Annals of Neurology, vol. 83, no. 6, pp. 1147-1161, 2018.

[41] S. K. Tankou, K. Regev, B. C. Healy et al., "Investigation of probiotics in multiple sclerosis," Multiple Sclerosis, vol. 24, no. 1 , pp. 58-63, 2018.
[42] F. Cignarella, C. Cantoni, L. Ghezzi et al., "Intermittent fasting confers protection in CNS autoimmunity by altering the gut microbiota," Cell Metabolism, vol. 27, no. 6, pp. 12221235.e6, 2018.

[43] J. Rodríguez-Morató, N. R. Matthan, J. Liu, R. de la Torre, and C.-Y. O. Chen, "Cranberries attenuate animal-based dietinduced changes in microbiota composition and functionality: a randomized crossover controlled feeding trial," The Journal of Nutritional Biochemistry, vol. 62, pp. 76-86, 2018.

[44] A. Koh, F. De Vadder, P. Kovatcheva-Datchary, and F. Backhed, "From dietary Fiber to host physiology: shortchain fatty acids as key bacterial metabolites," Cell, vol. 165, no. 6, pp. 1332-1345, 2016.

[45] G. den Besten, K. van Eunen, A. K. Groen, K. Venema, D. J. Reijngoud, and B. M. Bakker, "The role of shortchain fatty acids in the interplay between diet, gut microbiota, and host energy metabolism," Journal of Lipid Research, vol. 54, no. 9, pp. 2325-2340, 2013.

[46] L. Hoyles, T. Snelling, U. K. Umlai et al., "Microbiome-host systems interactions: protective effects of propionate upon the blood-brain barrier," Microbiome, vol. 6, no. 1, p. 55, 2018.

[47] S. M. Matt, J. M. Allen, M. A. Lawson, L. J. Mailing, J. A. Woods, and R. W. Johnson, "Butyrate and dietary soluble fiber improve neuroinflammation associated with aging in mice," Frontiers in Immunology, vol. 9, p. 1832, 2018.

[48] M. Lanza, M. Campolo, G. Casili et al., "Sodium butyrate exerts neuroprotective effects in spinal cord injury," Molecular Neurobiology, vol. 56, no. 6, pp. 3937-3947, 2019.

[49] M. Mizuno, D. Noto, N. Kaga, A. Chiba, and S. Miyake, "The dual role of short fatty acid chains in the pathogenesis of autoimmune disease models," PLoS One, vol. 12, no. 2, article e0173032, 2017.

[50] A. C. Chevalier and T. A. Rosenberger, "Increasing acetyl-CoA metabolism attenuates injury and alters spinal cord lipid content in mice subjected to experimental autoimmune encephalomyelitis," Journal of Neurochemistry, vol. 141, no. 5, pp. 721-737, 2017.

[51] Y. Wang, S. Begum-Haque, K. M. Telesford et al., "A commensal bacterial product elicits and modulates migratory capacity of CD39(+) CD4 T regulatory subsets in the suppression of neuroinflammation," Gut Microbes, vol. 5, no. 4, pp. 552-561, 2014.

[52] J. Ochoa-Repáraz, D. W. Mielcarz, L. E. Ditrio et al., “Central nervous system demyelinating disease protection by the human commensal Bacteroides fragilis depends on polysaccharide A expression," Journal of Immunology, vol. 185, no. 7, pp. 4101-4108, 2010.

[53] A. Heintz-Buschart, U. Pandey, T. Wicke et al., "The nasal and gut microbiome in Parkinson's disease and idiopathic rapid eye movement sleep behavior disorder," Movement Disorders, vol. 33, no. 1, pp. 88-98, 2018.

[54] J. R. Bedarf, F. Hildebrand, L. P. Coelho et al., "Functional implications of microbial and viral gut metagenome changes in early stage L-DOPA-naive Parkinson's disease patients," Genome Medicine, vol. 9, no. 1, p. 39, 2017.

[55] R. Araos, N. Andreatos, J. Ugalde, S. Mitchell, E. Mylonakis, and E. M. C. D'Agata, "Fecal microbiome among nursing home residents with advanced dementia and clostridium difficile," Digestive Diseases and Sciences, vol. 63, no. 6, pp. 1525-1531, 2018. 
[56] S. Liu, R. M. Rezende, T. G. Moreira et al., "Oral administration of miR-30d from feces of MS patients suppresses MSlike symptoms in mice by expanding Akkermansia muciniphila," Cell Host \& Microbe, vol. 26, no. 6, pp. 779794.e8, 2019, e778.

[57] F. E. Juul, K. Garborg, M. Bretthauer et al., "Fecal microbiota transplantation for Primary Clostridium difficile Infection," The New England Journal of Medicine, vol. 378, no. 26, pp. 2535-2536, 2018.

[58] P. Moayyedi, M. G. Surette, P. T. Kim et al., "Fecal microbiota transplantation induces remission in patients with active ulcerative colitis in a randomized controlled trial," Gastroenterology, vol. 149, no. 1, pp. 102-109.e6, 2015.

[59] D. Zhou, Q. Pan, F. Shen et al., "Total fecal microbiota transplantation alleviates high-fat diet-induced steatohepatitis in mice via beneficial regulation of gut microbiota," Scientific Reports, vol. 7, no. 1, p. 1529, 2017.

[60] A. Vrieze, E. Van Nood, F. Holleman et al., "Transfer of intestinal microbiota from lean donors increases insulin sensitivity in individuals with metabolic syndrome," Gastroenterology, vol. 143, no. 4, pp. 913-916.e7, 2012.

[61] D. W. Kang, J. B. Adams, A. C. Gregory et al., "Microbiota transfer therapy alters gut ecosystem and improves gastrointestinal and autism symptoms: an open-label study," Microbiome, vol. 5, no. 1, p. 10, 2017.

[62] S. Makkawi, C. Camara-Lemarroy, and L. Metz, "Fecal microbiota transplantation associated with 10 years of stability in a patient with SPMS," Neurol Neuroimmunol Neuroinflamm, vol. 5, no. 4, article e459, 2018.

[63] A. E. Hoban, R. M. Stilling, F. J. Ryan et al., "Regulation of prefrontal cortex myelination by the microbiota," Translational Psychiatry, vol. 6, no. 4, article e774, 2016.

[64] J. F. Cryan and T. G. Dinan, "Gut microbiota: microbiota and neuroimmune signalling-Metchnikoff to microglia," Nature Reviews. Gastroenterology \& Hepatology, vol. 12, no. 9, pp. 494-496, 2015.

[65] S. Stanisavljević, M. Dinić, B. Jevtić et al., "Gut microbiota confers resistance of albino Oxford rats to the inducvtion of experimental autoimmune encephalomyelitis," Frontiers in Immunology, vol. 9, p. 942, 2018. 